\title{
Curcumin in combination with piperine attenuates MPTP induced dopaminergic neuron injury in rats: Impact from behavioral to neurotransmitter alterations
}

\author{
Puneet Kumar ${ }^{1}$, Shamsher Singh ${ }^{2}$ \\ ${ }^{I}$ Department of Pharmaceutical Sciences and Technology, Maharaja Ranjit Singh Punjab Technical University, India, \\ ${ }^{2}$ ISF College of Pharmacy, Moga, India
}

MPTP is a neurotoxin which by regressing dopaminergic neurons produces Parkinson's like manifestations both in human and rodents. Curcumin, a diarylheptanoid has propitious antioxidant and neuroprotective activity but major complication is its poor oral bioavailability. The study was designed to elucidate the protective effect of curcumin in combination with piperine against MPTP induced PD like symptoms in rodents.

Material and Methods: Rats were administered MPTP (100 Mirco gram per micro liter bilaterally) for 3 days (1st, 4 th and $7 \mathrm{th})$. Curcumin (25 and $50 \mathrm{mg}$ per $\mathrm{kg}$ ) and combination of curcumin $(25 \mathrm{mg}$ per $\mathrm{kg}$ ) with piperine (2.5 $\mathrm{mg}$ per $\mathrm{kg})$ was administered daily for 21 days starting from the 7th day of 1st MPTP injection. All the behavioral observations (locomotor, rotarod, grip strength and narrow beam walk performance) were recorded at weekly intervals after MPTP treatment. On the 22nd day, the animals were sacrificed and the rat striatum was isolated for the estimation of biochemical parameters (LPO, GSH and nitrite), estimation of pro-inflammatory cytokine levels (IL-1 beta, IL-6 and TNF- alpha) and neurochemical analysis (DA, NE, 5- HT, GABA, Glutamate, DOPAC, HVA and 5-HIAA).

Results: The present finding had confirmed that chronic treatment with curcumin (for 14 days) produced beneficial effect against MPTP induced motor deficit, biochemical and neurochemical alterations in rats. Moreover combination of piperine $(2.5 \mathrm{mg} / \mathrm{kg})$ with curcumin $(25 \mathrm{mg} / \mathrm{kg})$ significantly potentiates the protective effect compared to curcumin alone treated group.

Conclusion: Oral administration of curcumin in combination with piperine had significantly ameliorated the behavioral and neurological alteration induced by MPTP through antioxidant and anti-inflammatory mechanism. 